Bull. Austral. Math. Soc.

$16 \mathrm{D} 90,16 \mathrm{~s} 90$

VoL. 47 (1993) [95-100]

\title{
PROJECTIVITY AND DUALITY IN A MORITA CONTEXT
}

\author{
ZHOU ZHENGPING
}

\begin{abstract}
The concepts of closed submodule, left and right annihilators are generalised and a necessary and sufficient condition on any Morita context is given so that the duality and projectivity between these sets exist. As a special case, we get Hutchinson's necessary and sufficient condition under a weaker hypothesis on the context.
\end{abstract}

Let $U$ be a left $R$-module and $S$ the endomorphism ring of $U$. In 1977 [6], under the assumption that $U$ is projective and contains a unimodular element, a proof was given that there is an order inverting bijection called duality, and an order preserving bijection called projectivity between the closed submodules of $U$ and the right, respectively, left annihilators in $S$ of subsets of $S$. In 1987 [3], in the more general setting of a Morita context $(R, U, V, S)$, under a much weaker hypothesis that $U_{S}$ is faithful and $(U, V)=R$, which is equivalent to $(R, U, V, S)$ being nondegenerate [1, Theorem 2] and $(U, V)=R$, Hutchinson proved that the duality and projectivity exist if and only if $U$ is torsionless. Since a projective module is torsionless, these results generalise the theorems in [6], and since necessary and sufficient conditions are given, these results are in some sense the best.

In this paper, we generalise the concepts of closed submodule, and left and right annihilators, and give a necessary and sufficient condition on any Morita context so that the duality and projectivity between these sets exist. As a special case, we get Hutchinson's necessary and sufficient condition under a weaker hypothesis on the context.

Throughout this paper, $R$ and $S$ are associative rings with identity, and $R$-Mod, $S$-Mod denote respectively the categories of unital left $R$-, and left $S$-modules. Modules, unless otherwise specified, are consistently left modules.

Recall for a Gabriel topology $\tau$, a module $M$ is called $\tau$-torsion if $\operatorname{Ann} R(m) \in \tau$ for every $m \in M$, and any module $M$ has a largest $\tau$-torsion submodule $T_{\tau}(M)$; a module $M$ is called $\tau$-free if $\operatorname{Ann}_{M}(\mathcal{A})=0$ for every $\mathcal{A} \in \tau$; a submodule $K$ of $M$ is called $\tau$-saturated if $M / K$ is $\tau$-free, and $T_{\tau}(M)$ is the smallest $\tau$-saturated submodule of $M$. For any submodule $K$ of $M, \bar{K}=\{m \in M, \mathcal{A} m \subseteq K$ for some $\mathcal{A} \in \tau\}$ is

Received 15th January, 1992

Copyright Clearance Centre, Inc. Serial-fee code: 0004-9729/93 \$A2.00+0.00. 
the smallest $\tau$-saturated submodule of $M$ containing $K$, and $K=\bar{K}$ if and only if $K$ is $\tau$-saturated. If $M$ is $\tau$-torsion and $N$ is $\tau$-free, then $\operatorname{Hom}(M, N)=0$. For more details about Gabriel topology or torsion theory, the reader is referred to $[8,2]$.

Recall that a Morita context $(R, U, V, S)$ consists of two rings $\mathrm{R}$ and $\mathrm{S}$, two bimodules ${ }_{R} U_{S, S} V_{R}$, and two bimodule homomorphisms $(-,-): U \otimes S V \longrightarrow R$ and $[-,-]: V \otimes_{R} U \longrightarrow S$ satisfying $u\left[v, u^{\prime}\right]=(u, v) u^{\prime}$ and $v\left(u, v^{\prime}\right)=[v, u] v^{\prime}$ for all $u, u^{\prime} \in U$ and $v, v^{\prime} \in V$ with the images being $I$ and $J$, respectively. $I$ and $J$ are both ideals and are called the trace ideals of the context.

Leu and Hutchinson [5], and Kašu [4] proved that for any context $(R, U, V, S)$, there is a lattice isomorphism between the lattice $G(R)$ of all Gabriel topologies containing $I$ on $R$-Mod, and the lattice $G(S)$ of all Gabriel topologies containing $J$ on $S$-Mod.

From now on, whenever we talk about $\tau$ or $\tau^{\prime}$, unless otherwise specified, we always mean that $\tau \in G(R), \tau^{\prime} \in G(S)$ being the corresponding one, denoted by $\left(\tau, \tau^{\prime}\right) \in(G(R), G(S))$.

TheOREM 1. $L_{S}: X \longmapsto L_{S}(X)=\left\{s \in S \mid s X \subseteq T_{\tau^{\prime}}(S)\right\}$, and $R_{S}: X \longmapsto$ $R_{S}(X)=\left\{s \in S \mid X s \subseteq T_{\tau^{\prime}}(S)\right\}$ form a Galois connection in the lattice of subsets of $S$. Therefore there exists an order inverting bijection between $\overline{L_{S}}=\left\{L_{S}(X) \mid X \subseteq S\right\}$ and $\overline{R_{S}}=\left\{R_{S}(X) \mid X \subseteq S\right\}$. Moreover $\overline{L_{S}}=\left\{X \subseteq S \mid X=L_{S} R_{S}(X)\right\}, \overline{R_{S}}=\{X \subseteq$ $\left.S \mid X=R_{S} L_{S}(X)\right\}$.

Proof: By a routine verification, $L_{S}$ and $R_{S}$ satisfy :

(1) $X_{1} \subseteq X_{2} \Longrightarrow L_{S}\left(X_{1}\right) \supseteq L_{S}\left(X_{2}\right)$ and $R_{S}\left(X_{1}\right) \supseteq R_{S}\left(X_{2}\right)$;

(2) $X \subseteq L_{S} R_{S}(X), X \subseteq R_{S} L_{S}(X)$ for any subset $X$ of $S$.

Theorem 2. $L_{U}: X \longmapsto L_{U}(X)$ and $R_{S}^{\prime}: U^{\prime} \longmapsto R_{S}^{\prime}\left(U^{\prime}\right)$ form a Galois connection between the lattice of subsets of $U$ and that of $S$, where $L_{U}(X)=\{u \in U \mid$ $\left.u X \subseteq T_{\tau}(U)\right\}, R_{S}^{\prime}\left(U^{\prime}\right)=\left\{s \in S \mid U^{\prime} s \subseteq T_{\tau}(U)\right\}$.

Therefore there exists an order inverting bijection between $\overline{L_{U}}=\left\{L_{U}(X) \mid X \subseteq S\right\}$ and $\overline{R_{S}^{\prime}}=\left\{R_{S}^{\prime}\left(U^{\prime}\right) \mid U^{\prime} \subseteq U\right\}$. Moreover $\overline{L_{U}}=\left\{U^{\prime} \subseteq U \mid U^{\prime}=L_{U} R_{S}^{\prime}\left(U^{\prime}\right)\right\}, \overline{R_{S}^{\prime}}=$ $\left\{X \subseteq S \mid X=R_{S}^{\prime} L_{U}(X)\right\}$.

Proof: This is also a routine verification as the previous theorem.

The following lemma will be used frequently in the sequel.

LEMma 3. (1) $[V, u] \subseteq T_{\tau^{\prime}}(S)$ if and only if $u \in T_{\tau}(U)$;

(2) $\quad U s \subseteq T_{\tau}(U)$ if and only if $s \in T_{\tau^{\prime}}(S)$.

Proof: Let $s \in T_{\tau^{\prime}}(S), \mathcal{B} \in \tau^{\prime}$ such that $\mathcal{B}_{s}=0$; then $U B s=0$. Consider

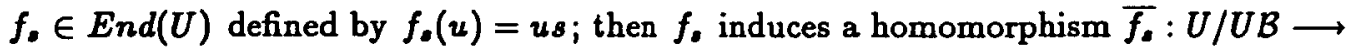


$U / T_{\tau}(U)$. But $U / U B$ is $\tau$-torsion by Lemma 2.1 in [9], and $U / T_{\tau}(U)$ is $\tau$-free, therefore $\bar{f}_{s}=0$; that is, $f_{o}(U)=U_{s} \subseteq T_{r}(U)$. So $U T_{r^{\prime}}(S) \subseteq T_{r}(U)$.

If $u \in T_{\tau}(U), \mathcal{A} \in \tau$ such that $\mathcal{A} u=0$, then $[V, \mathcal{A} u]=[V \mathcal{A}, u]=0$. Consider $f_{u} \in \operatorname{Hom}(V, S)$ defined by $f_{u}(v)=[v, u]$; then $f_{v}$ induces a homomorphism $\bar{f}_{u}$ : $V / V \mathcal{A} \longrightarrow S / T_{\tau}(S)$. But $V / V \mathcal{A}$ is $\tau^{\prime}$-torsion by Lemma 2.1 in $[\theta]$, and $S / T_{\tau}(S)$ is $\tau^{\prime}$-free, therefore $\overline{f_{w}}=0$, that is, $f_{u}(V)=[V, u] \subseteq T_{\tau^{\prime}}(S)$. So $\left[V, T_{\tau}(U)\right] \subseteq T_{r^{\prime}}(S)$.

Now if $U_{s} \subseteq T_{\tau}(U)$, then $[V, U]_{s}=\left[V, U_{s}\right] \subseteq\left[V, T_{\tau}(U)\right] \subseteq T_{\tau}(S)$. But $J=$ $[V, U] \in \tau^{\prime}$, and $T_{r^{\prime}}(S)$ is $\tau^{\prime}$-saturated, therefore $s \in T_{r^{\prime}}(S)$. Similarly, if $[V, u] \subseteq$ $T_{\tau^{\prime}}(S)$, then $(U, V) u=U[V, u] \subseteq U T_{\tau^{\prime}}(S) \subseteq T_{\tau}(U)$, and we get $u \in T_{\tau}(U)$.

THEOREM 4. $\overline{R_{S}^{\prime}}=\overline{R_{S}}$.

Proof: We shall show $R_{S}^{\prime}\left(U^{\prime}\right)=R_{S}\left(\left[V, U^{\prime}\right]\right)$, and $R_{S}(X)=R_{S}^{\prime}(U X)$ for any $U^{\prime} \subseteq U, X \subseteq S$.

First $s \in R_{S}^{\prime}\left(U^{\prime}\right) \Longleftrightarrow U^{\prime} s \subseteq T_{\tau}(U) \Longleftrightarrow\left[V, U^{\prime}\right]_{s}=\left[V, U^{\prime} s\right] \subseteq T_{\tau}(S)$ (by Lemma 3) $\Longleftrightarrow s \in R_{S}\left(\left[V, U^{\prime}\right]\right)$.

Secondly $s \in R_{S}^{\prime}(U X) \Longleftrightarrow U X_{s} \subseteq T_{\tau}(U) \Longleftrightarrow X_{s} \subseteq T_{\tau^{\prime}}(S)$ (by Lemma 3) $\Longleftrightarrow s \in R_{S}(X)$.

Now letting $F\left(U^{\prime}\right)=\left\{s \in S \mid U_{s} \subseteq U^{\prime}\right\}$, we have the following significant result.

Theorem 5. (1) The map $F$ above forms an order preserving bijection between $\overline{L_{U}}$ and $\overline{L_{S}}$;

(2) The map $G=R_{S} F$ forms an order inverting bijection between $\overline{L_{U}}$ and $\overline{R_{S}}$.

Proof: By Theorems 2 and 4, it remains to show that $R_{S}^{\prime}=R_{S} F$, and $L_{S} R_{S}^{\prime}=$ $\boldsymbol{F}$.

If $U^{\prime} \in \overline{L_{U}}$, and $s \in R_{S}^{\prime}\left(U^{\prime}\right)$, then $U F\left(U^{\prime}\right) s \subseteq U^{\prime} s \subseteq T_{\tau}(U)$ and so $F\left(U^{\prime}\right) s \subseteq$ $T_{\tau^{\prime}}(S)$ (by Lemma 3), and so $s \in R_{S} F\left(U^{\prime}\right)$. Conversely, if $F\left(U^{\prime}\right) s \subseteq T_{\tau^{\prime}}(S)$, then since it is easy to see that $U^{\prime} \in \overline{L_{U}}$ is a submodule of $U$ and $\left[V, U^{\prime}\right] \subseteq F\left(U^{\prime}\right)$, $\left[V, U^{\prime} s\right]=\left[V, U^{\prime}\right]_{s} \subseteq F\left(U^{\prime}\right) s \subseteq T_{\tau^{\prime}}(S)$. We have that $U^{\prime} s \subseteq T_{\tau}(U)$ by Lemma 3 , that is, $R_{S} F\left(U^{\prime}\right) \subseteq R_{S}^{\prime}\left(U^{\prime}\right)$.

Since $F\left(U^{\prime}\right) \in \overline{L_{S}}, L_{S} R_{S}^{\prime}=L_{S} R_{S} F=F$ by Theorem 1 .

Let us look at the special case where $\tau=\tau_{I}$ and $\tau^{\prime}=\tau_{J}$, (the topologies determined by the trace ideals $I$ and $J$ ), and where $T_{\tau}(U)=T_{r^{\prime}}(S)=0$ (which is equivalent to $(R, U, V, S)$ being nondegenerate, that is, $U_{S}$ is faithful and $[V, u] \neq 0$ whenever $0 \neq u \in U)$.

Let $K \subseteq U, X \subseteq S, A n n_{S} K=\{s \in S \mid K s=0\}, l(X)=\{s \in S \mid s X=0\}$, $r(X)=\{s \in S \mid X s=0\}$ and $A n n U X=\{u \in U \mid u X=0\}$. Let (see [3]) 


$$
\begin{aligned}
C_{A} & =\left\{K \subseteq U \mid K=A n_{U} A n_{S} K\right\} \\
\mathcal{L} & =\{X \subseteq S \mid X=\operatorname{lr}(X)\} \\
\mathcal{R} & =\{X \subseteq S \mid X=\operatorname{rl}(X)\}
\end{aligned}
$$

then it is clear that $\overline{L_{U}}=C_{A}, \overline{L_{S}}=\mathcal{L}$, and $\overline{R_{S}}=\mathcal{R}$, so we have

Corollary 6. If $(R, U, V, S)$ is nondegenerate, and $\tau=\tau_{I}, \tau^{\prime}=\tau_{J}$, then

(1) $F: C_{A} \longrightarrow \mathcal{L}$ is an order preserving bijection;

(2) $G: C_{A} \longrightarrow \mathcal{R}$ is an order inverting bijection.

In $\left[3\right.$, Theorem 3], the same result is proven with the assumption that $U_{S}$ is faithful and $u \in I u$ for all $u \in U$, which is much weaker than the existence of a unimodular element [6] and also weaker than $I=R$, but stronger than our assumption of the nondegeneracy here.

To see this, first we should mention a fact in [7, Proposition 1] that $M$ is $\tau$-free, where $\tau$ is determined by a two sided ideal $I$, if and only if $A n n_{M}(I)=0$. Now if $I u=0$, then $0=u \in I u=0$, so $U$ is $\tau_{I}$-free and $T_{\tau}(U)=0$. If $J_{s}=0$, then $U J_{s}=I U_{s}=0$, but $U$ is $\tau_{I}$-free, so we have $U_{s}=0$. However $U_{S}$ is faithful, so $s=0$, and $s S$ is $\tau_{J}$-free and $T_{\tau^{\prime}}(S)=0$.

In fact, if $X \in \mathcal{L}$, it is easy to check that $F^{-1}(X)=\overline{U X}$, while in [3] it is defined to be $U X$, and the stronger condition that $u \in I u$ for all $u \in U$ just makes $U X=\overline{U X}$ for all $X \in \mathcal{L}$. However, this is not necessary in establishing the desired bijection.

Let $K \subseteq U, K^{\perp}=\left\{v \in V \mid(K, v) U \subseteq T_{\tau}(U)\right\}, K^{\perp \perp}=\left\{u \in U \mid\left(u, K^{\perp}\right) U \subseteq\right.$ $\left.T_{\tau}(U)\right\}, \mathcal{C}=\left\{K \subseteq U \mid K=K^{\perp \perp}\right\}, \mathcal{T}=\left\{u \in U \mid(u, V) U \subseteq T_{\tau}(U)\right\}$; then we have:

ThEOREM 7 . (1) $\mathcal{C} \subseteq \overline{L_{U}}$;

(2) $T_{r}(U) \in \overline{L_{U}}$;

(3) $\mathcal{T}$ is $\tau$-saturated, therefore $T_{\tau}(U) \subseteq \mathcal{T}$;

(4) $T \subseteq T_{\tau}(U)^{\perp \perp}$.

Proof: (1) Let $K \in \mathcal{C}$; clearly $K \subseteq L_{U} R_{S}^{\prime}(K)$. Since $K\left[K^{\perp}, U\right]=\left(K, K^{\perp}\right) U \subseteq$ $T_{\tau}(U),\left[K^{\perp}, U\right] \subseteq R_{S}^{\prime}(K)$ and for any $u \in L_{U} R_{S}^{\prime}(K), u\left[K^{\perp}, U\right]=\left(u, K^{\perp}\right) U \subseteq T_{\tau}(U)$. Therefore $u \in K^{\perp \perp}=K$ and $K \supseteq L_{U} R_{S}^{\prime}(K)$. Hence $K=L_{U} R_{S}^{\prime}(K)$ and $K \in \overline{L_{U}}$.

(2) It is easy to check that $T_{\tau}(U)=L_{U} R_{S}^{\prime}(0)$.

(3) Clearly $\mathcal{T}$ is a submodule of $U$. It is also $\tau$-saturated. To see this let $\mathcal{A} \in \tau$, and $u \in U$ such that $\mathcal{A} u \subseteq \mathcal{T}$. Then $\mathcal{A}(u, V) U=(\mathcal{A} u, V) U \subseteq$ $T_{\tau}(U)$. For any $(u, v) u^{\prime} \in(u, V) U, \mathcal{A}(u, v) u^{\prime} \subseteq T_{\tau}(U)$, and therefore $(u, v) u^{\prime} \in T_{\tau}(U)$ since $\overline{T_{\tau}(U)}=T_{\tau}(U)$. Hence $(u, V) U \subseteq T_{\tau}(U), u \in \mathcal{T}$ and $\mathcal{T}=\overline{\mathcal{T}}$ 
(4) Let $u \in \mathcal{T}$, then $\left(u, T_{\tau}(U)^{\perp}\right) \subseteq(u, V)$, so $\left(u, T_{\tau}(U)^{\perp}\right) U \subseteq(u, V) U \subseteq$ $T_{r}(U)$ and therefore $u \in T_{\tau}(U)^{\perp \perp}$.

If the context is nondegenerate, ${ }_{R} U$ is faithful, and $\tau=\tau_{I}$, then (1) is exactly Theorem 4, (c) in [3].

THEOREM 8. $T=T_{\tau}(U)$ if and only if $\overline{L_{U}}=\mathcal{C}$.

Proof: It remains to show that $\overline{L_{U}} \subseteq \mathcal{C}$ if and only if $\mathcal{T}=T_{\tau}(U)$. If $\mathcal{T}=T_{\tau}(U)$, $K=L_{U} R_{S}^{\prime}(K) \in \overline{L_{U}}$, then clearly $K \subseteq K^{\perp \perp}$ by the definition. On the other hand, $K R_{S}^{\prime}(K) \subseteq T_{\tau}(U)=\mathcal{T}$ and so $\left(K R_{S}^{\prime}(K), V\right) U=\left(K, R_{S}^{\prime}(K) V\right) U \subseteq T_{\tau}(U)$. It follows that $R_{S}^{\prime}(K) V \subseteq K^{\perp}$, and so $\left(K^{\perp \perp}, R_{S}^{\prime}(K) V\right) U=\left(K^{\perp \perp} R_{S}^{\prime}(K), V\right) U \subseteq T_{\tau}(U)$. It follows that $K^{\perp \perp} R_{S}^{\prime}(K) \subseteq T=T_{\tau}(U)$, and so $K^{\perp \perp} \subseteq L_{U} R_{S}^{\prime}(K)=K$. So $K^{\perp \perp}=K$ and $K \in \mathcal{C}$.

Conversely suppose that $\mathcal{T} \neq T_{\tau}(U)$. Note that $T_{\tau}(U) \subseteq \mathcal{T}, T_{\tau}(U) \in \overline{L_{U}}$ and $T_{r}(U)^{\perp \perp} \supseteq \mathcal{T}$ by Theorem 7 (3), (2) and (4). Hence $T_{r}(U) \neq T_{\tau}(U)^{\perp \perp}$, that is, $T_{r}(U) \notin \mathcal{C}$.

This result contains Theorems 5,6 , and 10 in [3] as special cases when the context is nondegenerate, or both nondegenerate and ${ }_{R} U$ faithful, and $\tau=\tau_{I}$.

Combining Theorems 8, 5 and Corollary 7 (1), we get

Theorem 9. For any Morita context $(R, U, V, S)$, the following are equivalent:

(1) the duality between $\mathcal{C}$ and $\overline{R_{S}}$ exists;

(2) the projectivity between $\mathcal{C}$ and $\overline{L_{S}}$ exists;

(3) $T_{\tau}(U)=\mathcal{T}$.

This contains Corollary 7 in [3] as a special case. In fact, we have

Corollary 10. If $(R, U, V, S)$ is nondegenerate and ${ }_{R} U$ is I-faithful, that is, $i U \neq 0$ whenever $0 \neq i \in I$, then the following are equivalent;

(1) the duality between $C_{P}$ and $\mathcal{R}$ exists;

(2) the projectivity between $C_{P}$ and $\mathcal{L}$ exists;

(3) $\mathcal{T}=0$.

Here $C_{P}=\left\{K \subseteq U \mid K=K^{* *}\right\}, K^{*}=\{v \in V \mid(K, v)=0\}$ and $K^{* *}=\{u \in U \mid$ $\left.\left(u, K^{*}\right)=0\right\}$.

Proof: Under this assumption, $C_{P}=\mathcal{C}, \overline{R_{S}}=\mathcal{R}, \overline{L_{S}}=\mathcal{L}$ and $T_{\boldsymbol{r}}(U)=0$.

An element in $C_{P}$ is called a closed submodule of $U$ in [3]. If $V=H o m(U, R)$, then $\mathcal{T}=0$ if and only if $U$ is torsionless, and since $I=R$ implies ${ }_{R} U$ is $I$-faithful we get Hutchinson's result [3] under a weaker assumption as a special case. 


\section{REFERENCES}

[1] S.A. Amitsur, 'Rings of quotients and Morita contexts', J. Algebra 17 (1971), 273-298.

[2] J.S. Golan, Torsion theories (Longman Scientific and Technical, 1986).

[3] J.J. Hutchinson, 'Endomorphism rings of torsionless modules', Comm. Algebra 15 (1987), 1921-1927.

[4] A.I. Kašu, 'Morita contexts and torsions of modules', Math. Zametki. T. 28 (1980), 491-499.

[5] H.M. Leu and J.J. Hutchinson, 'Kernel functors and quotient rings', Bull. Inst. Math. Acad. Sinica 5 (1977), 145-155.

[6] B.R. McDonald, 'Endomorphism rings of infinitely generated projective modules', J. $A L$ gebra 45 (1977), 69-82.

[7] B.J. Müller, 'The quotient category of a Morita context', J. Algebra 28 (1974), 389-407.

[8] B. Stenström, Rings of quotients (Springer-Verlag, Berlin, Heidelberg, New York, 1975).

[9] Z. Zhou, 'Equivalence and duality of quotient categories', J. Algebra 143 (1991), 144-155.

Department of Mathematics

University of lowa

Iowa City IA 52242

United States of Americs 